\title{
Electro-Optic Near Field Imaging of High-Power RF/Microwave Transistors in Plastic Packages
}

\author{
Jonas Urbonas*, Frederik Vanaverbeke ${ }^{\dagger}, K_{e v i n} \mathrm{Kim}^{\dagger}$, Peter H. Aaen* \\ ${ }^{*}$ University of Surrey, Advanced Technology Institute, Department of Electrical and Electronic Engineering, Guildford, UK \\ ${ }^{\dagger}$ NXP Semiconductor, Chandler, AZ, USA
}

\begin{abstract}
In this paper, through-plastic vector E-field measurements of an LDMOS transistor in an over-molded plastic package are presented. The measurement system uses a commercially-available electro-optic system connected to an NVNA with a comb generator to non-invasively measure the phase-coherent multi-harmonic E-fields. The device is measured in a load-pull measurement system, which is used to present optimal source and load impedances to the transistor during the multi-harmonic E-field measurements. All three E-field components are measured at the fundamental $(2.2 \mathrm{GHz})$ and two harmonics at $P_{1 d B}=53.2 \mathrm{dBm}$.

Index Terms-Electro-optic probing, LDMOS, near-field imaging, power amplifier
\end{abstract}

\section{INTRODUCTION}

Microwave power transistors used in high power amplifiers (PAs), as shown in Fig. 1, are often the most expensive part of a wireless infrastructure base-station and the majority of this cost is due to the package. High-frequency plastic packaging technology is now in wide-spread use for highvolume manufacturing as it offers significant cost reductions as compared with air-cavity metal-ceramic packages.

Electric field probing on semiconductor devices has been performed over the last several decades but has recently seen a resurgence to examine the complicated internal operation of high-power packaged transistors. Knowledge of the E-fields within the package allows designers to, fault-find [1], [2], and to characterize the distributed behavior, and the affects of the various active and passive constituent components of the packaged transistor [3], [4]. These measurements allow the probing of the electromagnetic waves inside the packaged transistor and this has revealed significant insight into the overall device behavior and voltage distribution non-uniformities across the large periphery transistors [1], [2], [5].

Coaxial cable based assemblies, where a protruding center conductor acts as a monopole antenna, are sensitive to the normal E-field component and they have been used to probe the fields within air-cavity metal-ceramic packaged transistors [4], [6], [7]. These probes rely on capacitive coupling between the probe and the metalization of the device-under-test [8].

Recently, electro-optic (EO) measurement methods have been used to measure the vector E-fields within these devices [5], [9]. This measurement technique relies on the E-field induced laser light polarization change in a birefringent dielectric material, known as Pockels effect. A small birefringent crystal is mounted to the end of a polarization maintaining fiber optic cable as shown in Fig. 2. The laser passes through the crystal and hits a Brag reflector at the opposite side of

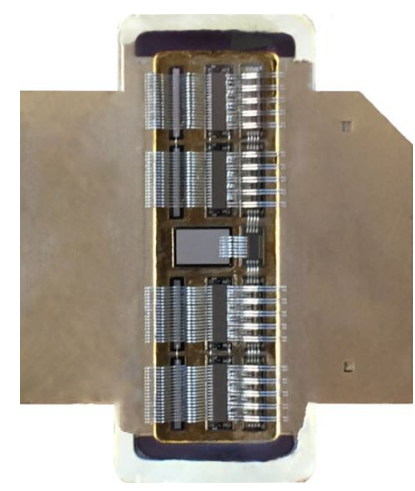

(a)

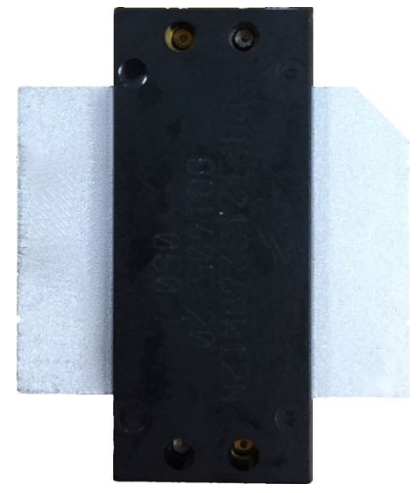

(b)
Fig. 1. A 260-W LDMOS a air-cavity metal-ceramic package with its lid removed (a), and in an over-molded plastic package (b). Gate lead is on the left side of the device, and the drain lead is on the right.

the crystal which reflects the beam back into the optical mainframe. From the polarization change measured in the reflected beam the magnitude and phase of the E-field at the measurement point is determined. Using probes, with crystals mounted in different directions, the $E_{x}, E_{y}$, and $E_{z}$ field components are measured, which is very useful for devices that are encapsulated in plastic where physical access to the internal structure of the device is restricted.

In this paper, we present an electro-optic probe-based Efield measurement above a $260-\mathrm{W}$ plastic packaged LDMOS power transistor in the load-pull bench for the first time. The paper is structured as follows: Section II describes the combined electro-optic NVNA measurement system used to measure the electric-fields above a high-power transistor. Section III describes the device geometry and presents measurement results that show the operation of the transistor. Finally, Section IV concludes the paper.

\section{Pulsed NVNA EO Measurement Setup}

The measurement setup, as fully described in [5] and shown in Fig. 3, uses a commercially available NeoScan ${ }^{\mathrm{TM}}$ electrooptic (EO) measurement system from EMAG Technologies Inc. ${ }^{~}$. Vector electric-field (E-field) measurements with amplitudes of $1 \mathrm{~V} / \mathrm{m}-2 \mathrm{MV} / \mathrm{m}$, and $20 \mathrm{MHz}-20 \mathrm{GHz}$ bandwidth are performed using external $\mathrm{Bi}_{12} \mathrm{SiO}_{20}$ (BSO) all-dielectric optical fiber-coupled electro-optic probes, as shown in Fig. 2. To enable multi-harmonic phase-coherent

\footnotetext{
${ }^{1}$ www.emagtech.com
} 


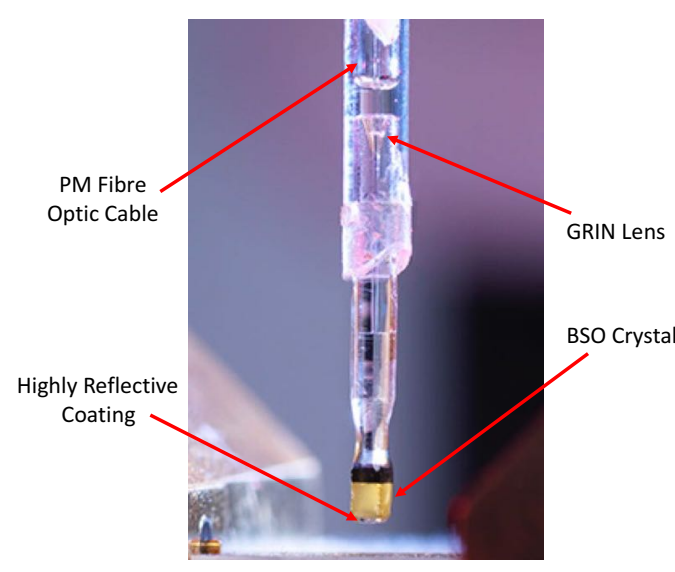

Fig. 2. Structure of a dielectric fiber-coupled electro-optic probe used during the vector E-field measurements.

electric field measurements the system is connected to a Keysight Technologies N5247A PNA-X 67 GHz non-linear vector network analyzer (NVNA) which uses an external comb generator as a phase reference. During the active device characterization, load-pull measurements are enabled by two Maury MT982BL01 impedance tuners, which are used to present various source and load impedances to the device under test (DUT). A 2-D translation stage is employed to move the probe above the DUT enabling E-field measurements over a scanned area. The measurement set-up is controlled by a custom measurement software implemented using National Instruments ${ }^{\mathrm{TM}}$ LabVIEW.

The measured phase-coherent multi-harmonic E-fields can be used to reconstruct time-domain E-fields above the device under test (DUT). This is achieved through a pixel-by-pixel time-domain summation of all harmonics of the distributed Efields measured above the DUT [5]. Measured time-domain electric fields are animated and provide significantly more information compared to the the static pictures. These animations will be presented at the conference and for the submission evaluation can be viewed here: https://goo.gl/pde2bJ.

For absolute time-domain traveling wave measurements, a calibration of the combined NVNA and EO system is performed. The calibration algorithm, presented in [5] and [8], uses a calibrated frequency-domain NVNA port measurement of a time-domain waveform to normalize the measured raw electro-optic signal as shown in Fig. 4. To achieve this, a microstrip transmission line is de-embedded to its middle using a thru-reflect-line (TRL) calibration. This results in having the same reference plane for both NVNA port-based, and electro-optic measurements. After the calibration, the EO probe can be used to non-invasively measure absolute timedomain waveforms anywhere above the DUT. As Pockels effect used for the E-field measurements has a linear relationship between the electric field induced light polarization change and the E-field strength sensed by the EO crystal, the relative distributed E-field measurement results presented in this paper are normalized to their own maximum. The absolute E-field

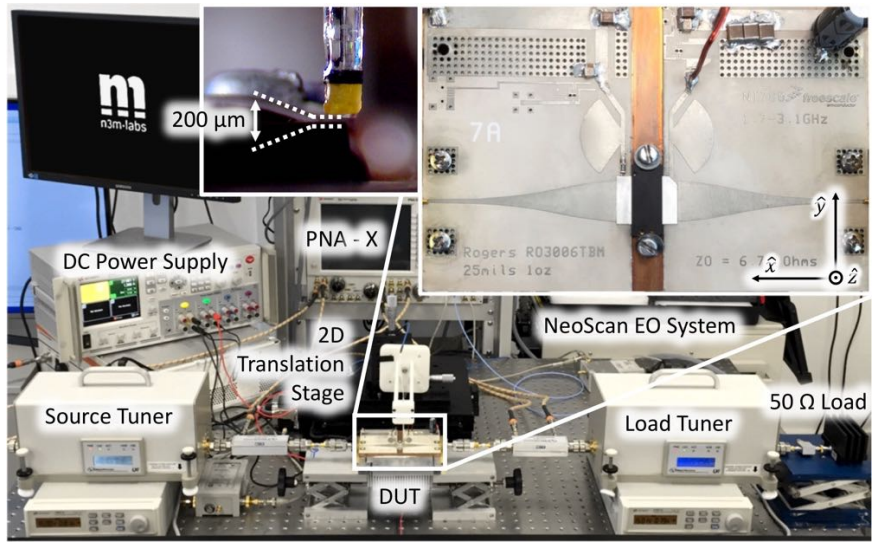

Fig. 3. Setup of the combined NVNA, electro-optic and load-pull measurement system used for the multi-harmonic phase-coherent vector E-field measurements.

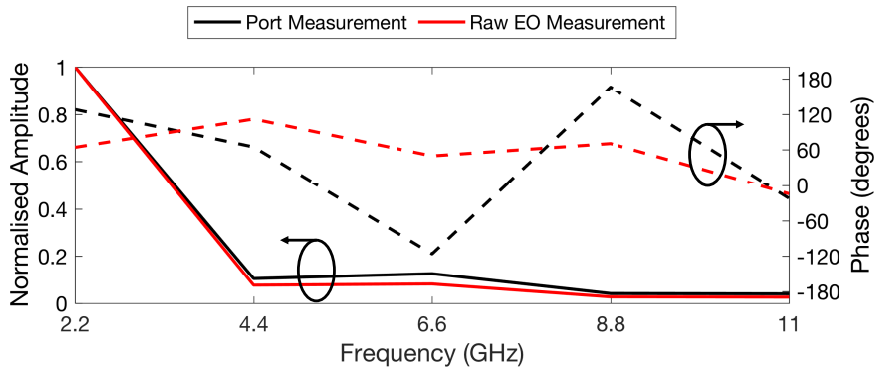

Fig. 4. Calibrated NVNA port (black), and raw EO signal (red) frequency domain data used during the signal calibration.

calibration can be obtained by employing a $\mu$-TEM cell [10].

\section{Device And Measurement Results}

The 260-W large-periphery LDMOS transistor has 4 transistor dies connected in parallel and is shown in Fig. 1, similar to the air-cavity metal-ceramic version. The matching network of the transistor is composed of arrays of parallel bonding wires, metal-oxide-semiconductor (MOS) capacitors, and the package, and is designed to increase the input and output impedances of the device for easier matching on the printed circuit boards (PCB). The over-molded plastic packaged transistor was measured inside a load-pull test-fixture with a tapered impedance transformer with an impedance transformation ratio of $1: 0.135$, as shown in Fig. 3 .

A fundamental frequency load-pull measurement was performed to find the max $\mathrm{P}_{\text {out }}$ impedance point at $\mathrm{P}_{l d B}$ gain compression. The NVNA was configured to measure 3 harmonics of a $2.2 \mathrm{GHz}$ fundamental. The noise bandwidth of the NVNA was set to $500 \mathrm{~Hz}$, and the averaging was set to 5 . The measurement reference planes were at the ends of $3.5 \mathrm{~mm}$ connectors of the load-pull test-fixture. A full NVNA calibration was performed at the connector ends, including short-open-load-through (SOLT) vector receiver calibration, absolute phase calibration using a comb generator and an absolute power calibration using a power meter. The transistor was biased at $\mathrm{V}_{\mathrm{ds}}=28 \mathrm{~V}, \mathrm{I}_{\mathrm{ds}}=1.5 \mathrm{~A}, \mathrm{~V}_{\mathrm{gs}}=2.66 \mathrm{~V}$. The 

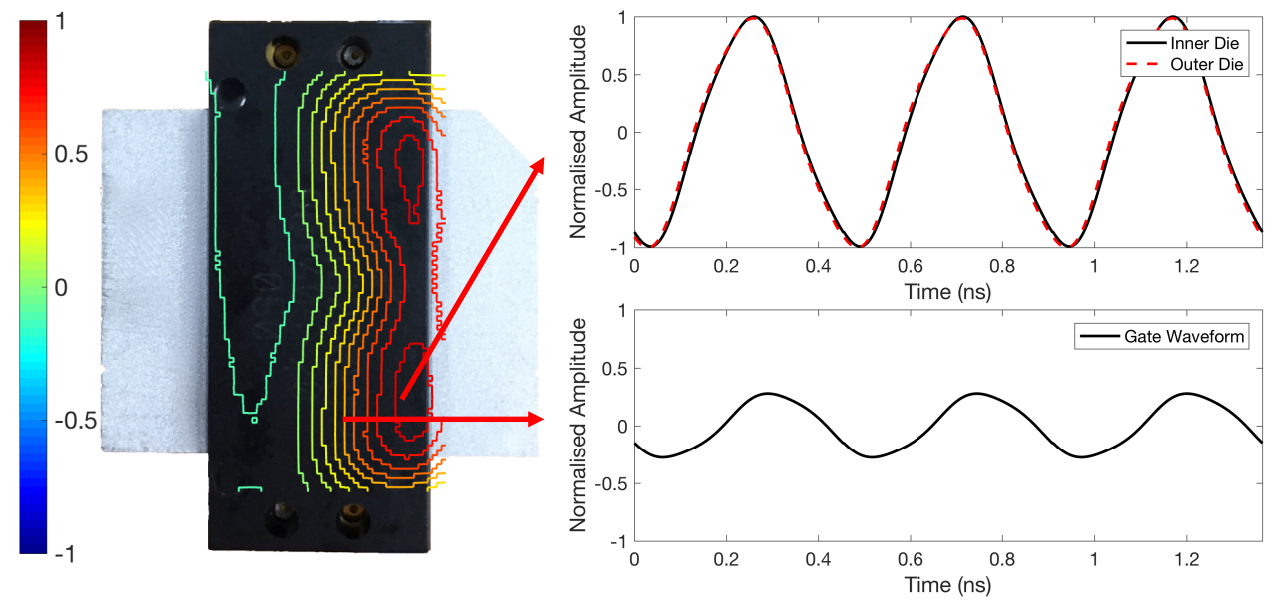

Fig. 5. Time-domain gate and drain waveforms reconstructed using the $\mathrm{E}_{\mathrm{z}}$-field measurement of 3 harmonics above a 260-W LDMOS transistor in plastic encapsulation. The waveforms reveal a small phase difference between the two adjacent transistor dies at the bottom half of the device.

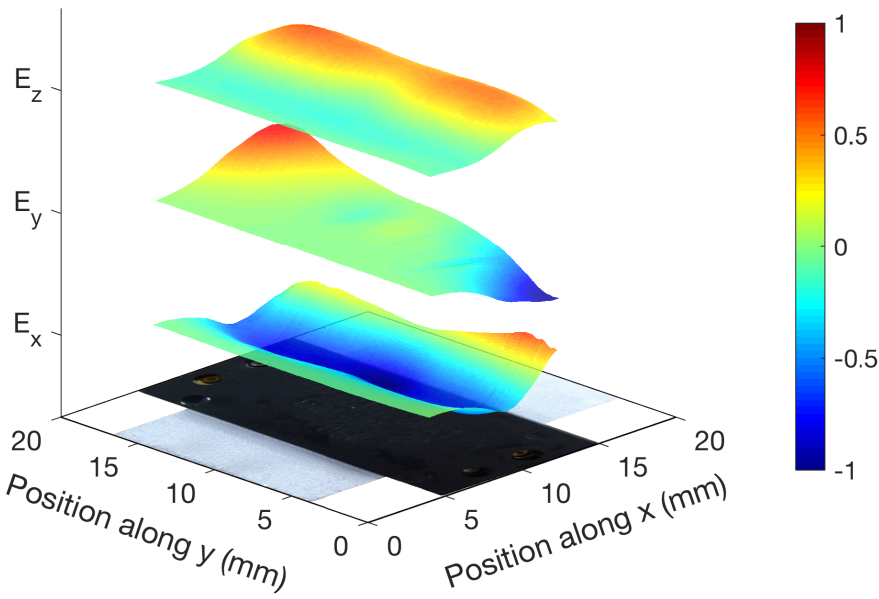

Fig. 6. Measured $E_{\mathrm{x}}, \mathrm{E}_{\mathrm{y}}$ and $\mathrm{E}_{\mathrm{z}}$ relative electric field distributions over a 260-W LDMOS transistor in an over-molded plastic package at a $2.2 \mathrm{GHz}$ fundamental.

input signal was a pulsed continuous wave with a pulse width of $10 \mu \mathrm{s}$ and a $100 \mu$ s period. The max $\mathrm{P}_{\text {out }}$ at $\mathrm{P}_{l d B}$ gain compression point was $53.2 \mathrm{dBm}$ at $\mathrm{P}_{\text {in }}=40.2 \mathrm{dBm}$.

The E-field measurements of the transistor were performed at the $\mathrm{P}_{I d B}$ gain compression point. The EO probe was positioned $200 \mu \mathrm{m}$ above the plastic package. The E-field scan area was $11 \times 18 \mathrm{~mm}$ with the step resolution of $0.1 \mathrm{~mm}$ on $x$ and $0.2 \mathrm{~mm}$ on $y$ axes for the $\mathrm{E}_{\mathrm{x}}$ component, $0.2 \mathrm{~mm}$ on $x$ and $0.1 \mathrm{~mm}$ on $y$ axes for the $\mathrm{E}_{\mathrm{y}}$ component, and $0.2 \mathrm{~mm}$ on both $x$ and $y$ axes for the $\mathrm{E}_{z}$ component measurements. The measurement results of all three vector E-field components at $2.2 \mathrm{GHz}$ fundamental are shown in Fig. 6. The E-field measurements reveal a strong gate-to-drain coupling in the $E_{x}$ component, no inter-die coupling in the $\mathrm{E}_{\mathrm{y}}$ component and an almost uniform $\mathrm{E}_{\mathrm{z}}$ component. This is expected from a normal device operation.

\section{CONCLUSION}

Multi-harmonic phase-coherent E-field measurements of a 260-W LDMOS transistor in an over-molded plastic packaging are presented. The combined electro-optic and NVNA measurement system used to obtain these measurements offers a unique approach to device characterization, operation analysis, and fault finding regardless of the device encapsulation. The obtained animated measurement data reveals a complex device behavior over the whole device operation period at the fundamental frequency, providing significantly more information as compared to static images.

\section{REFERENCES}

[1] N. Dehghan, S. Cripps, A. Porch, and J. Lees, "An Improved Electric Field Probe with Applications in High Efficiency PA Design and Diagnostics," in 81st Microw. Meas. Conf. (ARFTG), Seattle, WA, USA, 2013, pp. 1-4.

[2] R. Hou, M. Lorenzini, M. Spirito, T. Roedle, F. van Rijs, and L. C. N. de Vreede, "Nonintrusive Near-Field Characterization of Spatially Distributed Effects in Large-Periphery High-Power GaN HEMTs," IEEE Trans. Microw. Theory Techn., vol. 64, no. 11, pp. 4048-4062, Oct. 2016.

[3] S. C. Cripps and A. Porch, "An Active, Non-intrusive, High Resolution Microwave Field Probe with Applications in High Power RF Device and Circuit Design," in IEEE 11th Annu. Wirel. Microw. Technol. Conf. (WAMICON), Melbourne, FL, USA, 2010, pp. 1-4.

[4] R. Hou, M. Spirito, R. Heeres, F. van Rijs, and L. C. N. de Vreede, "Nonintrusive Near-field Characterization of Distributed Effects in Largeperiphery LDMOS RF Power Transistors," in IEEE MTT-S Int. Microw. Symp. (IMS), Pheonix, AZ, USA, 2015, pp. 1-3.

[5] J. Urbonas, K. Kim, F. Vanaverbeke, and P. H. Aaen, "An Electro-Optic Pulsed NVNA Load-Pull System for Distributed E-Field Measurements," IEEE Trans. Microw. Theory Techn., Submitted for review.

[6] N. Dehghan, A. Porch, S. C. Cripps, and P. H. Aaen, "A Novel High Resolution E-field Microscope System with Applications in HPA Diagnostics," in 78th Microw. Meas. Symp. (ARFTG), Tempe, AZ, USA, 2011, pp. 1-3.

[7] R. Hou, M. Spirito, J. Gajadharsing, and L. C. N. de Vreede, "Non-intrusive Characterization of Active Device Interactions in Highefficiency Power Amplifiers," in IEEE MTT-S Int. Microw. Symp. Dig. (IMS), Seattle, WA, USA, 2013, pp. 1-3.

[8] R. Hou, M. Spirito, F. V. Rijs, and L. C. N. d. Vreede, "Contactless Measurement of Absolute Voltage Waveforms by a Passive ElectricField Probe," IEEE Microw. Wireless Compon. Lett., vol. 26, no. 12, pp. 1008-1010, Nov. 2016 
[9] C. T. Rodenbeck, K. A. Peterson, C. E. Sandoval, K. Brakora, J. Thiesen, E. M. Russick, and R. A. Ortiz, "Electrooptic Inspection of Vector Leakage in Radiofrequency Multichip Modules," IEEE Trans. Electromagn. Compat., vol. 55, no. 6, pp. 1093-1099, Jul. 2013.

[10] N. W. Kang, J. S. Kang, D. C. Kim, J. H. Kim, and J. G. Lee, "Characterization Method of Electric Field Probe by Using Transfer Standard in GTEM Cell," IEEE Trans. Instrum. Meas., vol. 58, no. 4, pp. 1109-1113, Dec. 2008. 